\title{
Effects of curing time and filler concentration on curing and postcuring of urethane dimethacrylate composites: A microcalorimetric study
}

\author{
N. M. Mohsen, ${ }^{1}$ R. G. Craig, ${ }^{2}$ F. E. Filisko ${ }^{3}$ \\ ${ }^{1}$ Bioengineering Program, University of Michigan, Ann Arbor, Michigan 48109-1078 \\ ${ }^{2}$ School of Dentistry, University of Michigan, Ann Arbor, Michigan 48109-1078 \\ ${ }^{3}$ Department of Materials Science and Engineering, University of Michigan, Ann Arbor, Michigan 48109-1078
}

Received 22 April 1996; accepted 11 June 1997

\begin{abstract}
The isothermal enthalpy changes with time of a dental composite were examined by microcalorimetry to isolate the effects of different filler concentrations and curing times on chemical aging of these composites. Urethane dimethacrylate (UDMA) monomer, zirconia-silica (ZS) powder, and 3-methacryloxypropyltrimethoxysilane (MAPM) were used as organic and inorganic matrices, and a coupling agent, respectively. The composite was mixed in different ratios and cured by visible light. The enthalpy changes with time for 0, 15, 45, 75\% ZS-filled UDMA and 75\% MAPMsilanated ZS-filled UDMA cured for 13, 30, 90, 150, and $300 \mathrm{~s}$ were measured at $37.0^{\circ}, 57.0^{\circ}$, and $65.5^{\circ} \mathrm{C}$ until equilibrium. Increased curing time and filler concentration caused the excesss enthalpy changes $(\mathrm{dH})$ and their rate of change $(d H / d t)$ to increase with annealing time and apparent equilibrium was reached faster. In addition, $d H$ showed nonlinear dependence with the increase in filler concentration by showing a maxima for samples containing $25 \mathrm{wt} \%$ filler. Further, filler silanation caused $d H / d t$ to increase and required shorter times to reach apparent equilibrium. $d H$ also reached a minimum when samples contained silanated
\end{abstract}

filler, compared to composites containing unsilanated filler. It was concluded that the shorter curing time caused the occurrence of spontaneous densification, which facilitated continual resin curing; and longer curing time caused higher crosslinking of the organic phase. Moderate concentration of inorganic phase restricts the molecular motion of the surface layer of polymer onto filler particles, and the polymer is regarded as highly crosslinked, while a higher filler concentration forms aggregates that are covered by the polymer which causes a decrease in the molecular packing of the resin, and is reflected as low enthalpy values. Finally, silanation of the filler showed a highly endothermic reaction that is probably due to breaking and forming of bonds at the interface between the organic and the inorganic phases in the composites. (C) 1998 John Wiley \& Sons, Inc. J Biomed Mater Res, 40, 224-232, 1998.

Key words: chemical aging of UDMA; enthalpy changes of UDMA; microcalorimetry measurements; filler effects in composites on enthalpy changes; silane effects in composites on enthalpy changes

\section{INTRODUCTION}

In polymer-based composites, the properties of the organic matrix strongly influence the behavior of the composite material. The long-term stability of the matrix, therefore, is an important aspect of the composite's performance $^{1}$ and is highly dependent on the extent of the polymerization during the cure. For instance, inadequate cure and the presence of unreacted chemicals in the composite resin used in a highly reactive biological environment may lead to clinical problems such as slow degradation, color instability, poor durability, and loss of anatomic form. In addi-

Correspondence to: R. G. Craig

Contract grant sponsor: National Institute for Dental Research; Contract grant number: P50 DE 09296

(c) 1998 John Wiley \& Sons, Inc.

CCC 0021-9304/98/020224-09 tion, the presence of the unreacted monomer and oligomer leads to plasticization effects (lowering of the glass temperature), which causes changes in the physical as well as the mechanical properties.

Curing over long periods of time (chemical aging) in light-cured composites is caused by a continual conversion and polymerization of unreacted groups in the difunctional vinyl monomers. This reaction is a highly exothermic process, and the heat liberated tends to raise the local temperature and accelerate the curing reaction.

The mechanism of free radical polymerization of vinyl monomers can be divided into three stages: an initial stage; an intermediate stage in which the polymerization increases, defined as autoacceleration, and is known as the gel effect; and a final stage in which polymerization decreases as the monomer is depleted. In the end of the intermediate stage, the mobility of the system and the frequency of collisions decreases 
rapidly as the gel forms. In addition, since the frequency of termination reaction is a function of the rate of their meeting other growing chains, the proportion of the termination reaction is decreased, while that of addition reaction is increased. The increase in the addition reaction causes more heat to be liberated, and thus, the structure rapidly becomes more rigid and mobility decreases. The heat also increases the activity of the growing chains as well as the free radicals. This condition results in further densification of the structure and the formation of a glass. Upon further curing with time, diffusion of molecular segments of chains occurs, owing to spontaneous movement toward equilibrium (physical aging), further increasing crosslinking of chemically unreacted groups. These crosslinks cause further spontaneous relaxations to occur. Thus, physical aging and chemical aging are mutually dependent. $^{2}$

Many factors may have catalytic effects on curing of the resin such as impurities, unreactive raw materials, catalysts, monomeric species, and additives such as (chemical aging), and to investigate the effects of incorporation of filler on this process.

\section{MATERIALS AND METHODS}

\section{Composite system}

Urethane dimethacrylate (UDMA) (Esschem Co., Essington, PA) was used as a monomer system; its structural formula is given below. Zirconia-silica (ZS) with a surface area of $1.6 \mathrm{~m}^{2} / \mathrm{g}$ and an average diameter of $1.5 \mu \mathrm{m}$ (3M Dental Products, St. Paul, MN) was used as a filler. 3-Methacryloxypropyltrimethoxysilane (MAPM) (Huls America, Piscataway, NJ; formerly Petrarch Systems, Bristol, PA) was used as a coupling agent to silanate the filler. MAPM was selected because it has been shown by Craig and Mohsen ${ }^{7}$ to be one of the most effective silanating agents for increasing the dispersion and wetting behavior of the filler particles by the monomer, and has a carbon double bond to react with the monomer.

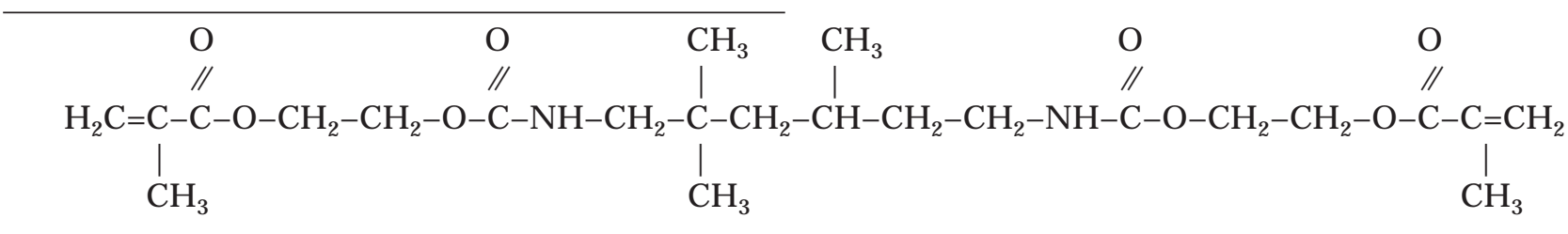

filler. The effect of these agents is specific for each resin system. For example, mineral fillers were found to have both positive and negative catalytic effects on the curing of resin. Both silica and E-glass can reduce the exotherm in an epoxy resin-aromatic amine, ${ }^{3}$ while silane coupling agents may give higher exotherms and provide composites with better mechanical properties and chemical resistance. ${ }^{4}$

Although determination of the curing process of thermosets, particularly beyond the gel stage, is very difficult, numerous methods have been used to investigate this process, such as chemical and spectrographic methods. However, these methods have limits and constraints in measuring the extent of the curing process with time, since the continual curing involves a minute evolution or absorption of heat over a long duration. To detect these small changes accurately with time, an instrument with high sensitivity and long-term stability is required. Therefore, a TianCalvet microcalorimeter was used in this study to monitor these changes continuously, since it has been proven to be suitable for these types of measurements. ${ }^{5,6}$

The purposes of this study were to investigate the isothermal enthalpy changes with time for a dental composite that was cured for different curing times

\section{Sample preparation}

The filler was silanated by depositing the silane from aqueous solution of $75 \%$ ethanol (190 proof) to $25 \%$ silane (weight ratio) as was described by Craig and Mohsen. ${ }^{7}$ Composites were formulated from UDMA, zirconia-silica, and MAPM-silanated zirconia-silica, using DL-camphoroquinone catalyst and 2-dimethylaminoethyl-methacrylate accelerator in concentrations described by Douglas et al. ${ }^{8}$ The amount of silane used to treat the filler was three times the minimum uniform coverage. ${ }^{7}$

The mixed composite paste was placed in Teflon discshaped molds ( $6.0 \mathrm{~mm}$ diameter $\times 3.5 \mathrm{~mm}$ thick) which were covered with glass to extrude excess material and prevent the formation of a surface-inhibited layer. The composite sample was made in two layers and each cured by visible light in a Triad II oven (Dentsply International, York, PA) for $13,30,90,150$, and $300 \mathrm{~s}$ at room temperature. The weight percentages of the unsilanated zirconia-silica in the composite samples were $0,15,45$, and $75 \mathrm{wt} \%$; it was $75 \mathrm{wt} \%$ for the MAPM-silanated zirconia-silica-filled UDMA samples.

\section{Enthalpy measurement}

The enthalpy changes were measured using a Tian-Calvet microcalorimeter (Setaram, Lyon, France). This instrument 
measures isothermally the heat evolution and absorption by continuously monitoring heat flow from and to the material. Three Tian-Calvet microcalorimeters were used, and a vertical cross section and the experimental assembly of the Tian-Calvet microcalorimeter are shown in Figure 1.

The microcalorimeter is connected to a temperature regulator (PRT 3000 RA; Setaram) for stabilizing the temperature of the thermostat for any chosen value between ambient and $200^{\circ} \mathrm{C}$ with a sensitivity and stability of $10^{-3 \circ} \mathrm{C}$. The apparatus is also connected to a nanovoltmeter (Model 181; Keithley), which measures of the calorimetric signal with a resolution of $10 \mathrm{nV}$. The microcalorimeter is interfaced with an automated data acquisition system based on a Macintosh II computer and Lab VIEW (International Instruments) software programs. The entire assembly is placed in an airconditioned room to stabilize the ambient temperature to $\pm 1^{\circ} \mathrm{C}$.

Because of the time delay required to reach thermal equilibrium between the sample and the calorimetric block, meaningful data cannot be obtained until some time after insertion of the sample, depending on the instrument temperature. Preliminary experiments were conducted to determine the temperatures and times needed for the resin to polymerize under heat, so the thermal equilibrium constraint could be accounted for. The UDMA resin was placed in glass tubes and kept in an oil bath at a constant high temperature. A glass rod was used to stir the resin constantly with a slow circular motion. The temperature at the time required for the resin to turn from a viscous liquid to an elastomer beyond the microcalorimetry equilibrium time was chosen as an instrument temperature.

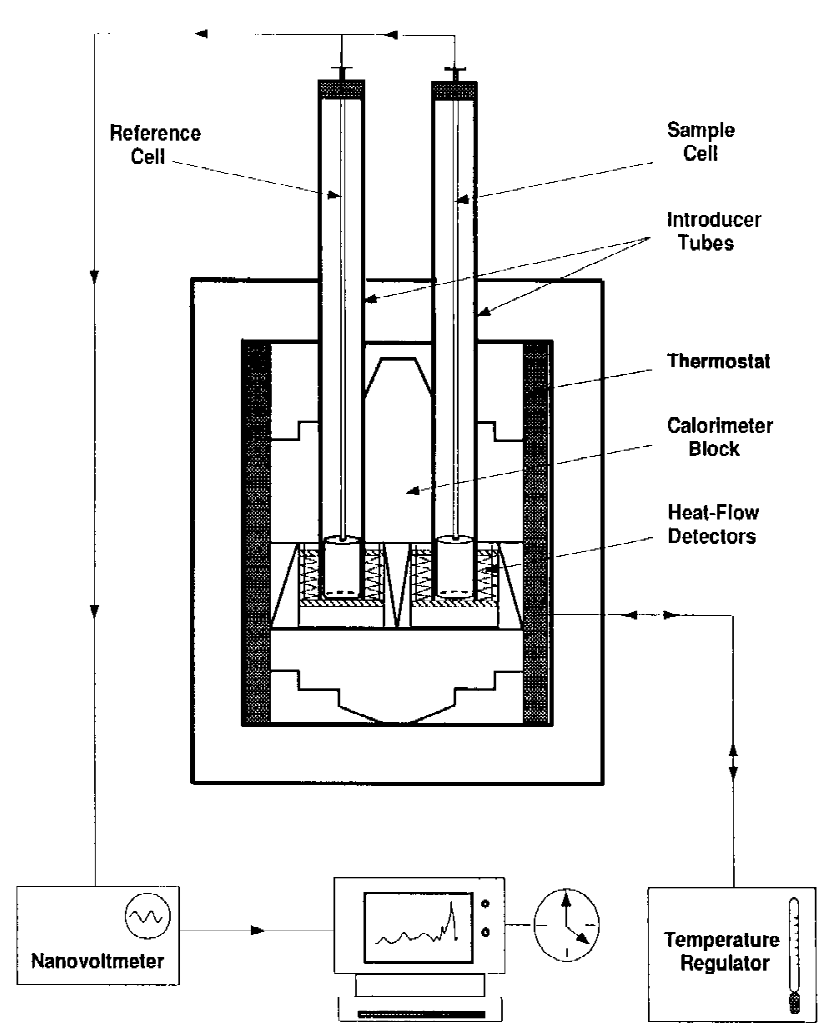

Figure 1. Tian-Calvet microcalorimeter cross-section and apparatus assembly.
Based on the preliminary results, the three microcalorimeters were set at temperatures below the $T_{g}$ for the entire investigation; one was set at $65.5^{\circ} \mathrm{C}$, another at $57.0^{\circ} \mathrm{C}$, and the third at $37.0^{\circ} \mathrm{C}$ to simulate body temperature. The thermal equilibrium for each instrument was determined by introducing equal amounts of ZS into both sample and reference cells and monitoring the time needed for the thermovoltage to level off. Thus, the thermal equilibrium for the instruments were $3.3,2.5$, and $0.5 \mathrm{~h}$ at $65.5^{\circ}, 57.0^{\circ}$, and $37.0^{\circ} \mathrm{C}$, respectively.

After reaching the temperature setting, the instruments were allowed to stabilize for at least a week before we conducted experiments. For each measurement, the sample cells, which were cylindrical in shape and made of stainless steel, were cleaned with soap and water and dried thoroughly. We introduced 1.5-2.5 g of specimen and a similar amount of reference material (ZS) into the sample and reference cell, respectively. Both cells were loaded into the calorimeter chambers simultaneously, the data acquisition program was invoked immediately, and measurements were started instantaneously. The sampling rate varied during the course of measurement with shorter intervals at short times and longer intervals at long times. The measurement was ended when the thermovoltage leveled off.

The measurements were recorded in the form of thermovoltage $(d E / d t)$ changes with time $(t)$, that were detected by the thermocouples. Recording of $(d E / d t)$ were taken every $200 \mathrm{~s}$ up to $10^{6} \mathrm{~s}$, after which they were taken every $60 \mathrm{~min}$ until the thermovoltage leveled off. The correlation between the measured thermovoltage and the actual rate of heat generated or absorbed by the samples was carried out by multiplying the $(d E / d t)$ values by the calibration factors. The calibration factors were determined by the following procedure: Two calibration cells, each with resistance of $1 \mathrm{k} \Omega$, that were supplied with the instrument were simultaneously inserted into the sample and reference chambers. After instrument equilibration, a known level of current was applied to the sample cell to generate a heat flow between the cell and the calorimetric block. When a steady state of thermovoltage power was reached, the thermovoltage readings also leveled off to reach a constant value. The height of the change of the thermovoltage that corresponded to the known current was recorded. A series of known current magnitudes were applied which corresponded to different power generations and resulted in different thermovoltage values. Thus, a linear regression curve was established to determine the correlation between the thermovoltage and the actual rate of heat generation. The slope of the regression line is the calibration factor $k$, which is given by

$$
k=\frac{R I^{2}}{E}
$$

where $R$ is the resistance of the calibration cell, $I$ is the magnitude of the applied current, and $E$ is the height of the thermovoltage between the two equilibrium states before and after the current application. For the microcalorimeters used in this study, the values of $k$ were $3.6364 \times 10^{-6}, 3.944$ $\times 10^{-6}$, and $4.055 \times 10^{-6} \mathrm{mcal} / \mathrm{s} \mathrm{nV}$.

The $d E / d t$ versus $t$ curves were converted into enthalpy changes $(d H / d t)$ versus $t$ curves by using the calibration factors. In $d H / d t$ versus $t$ curves, the baseline was determined 
by averaging the last 50 calorimetric points when the measured voltage became constant with time. The enthalpy changes $(d H)$ versus time were determined by integrating $d H / d t$ from the equilibrium time to the beginning of the results. The extent of the reaction or conversion as a function of time was determined by integrating the isothermal curves, using the relationship

$$
\text { Conversion }(\%)=\frac{100}{\Delta H_{T}} \int_{0}^{t} \frac{d H}{d t} d t
$$

where $\Delta H_{T}$ is total enthalpy change which is calculated from the integral of $d H / d t$ from $t=0$ (beginning of the measurement, i.e., when instrument thermal equilibrium is reached) to $t=\infty$ (end of measurement, i.e., when sample equilibrium is reached). The $d H / d t$ and $d H$ as a function of time were reported per weight of the polymer in the samples. The rates of enthalpy changes and enthalpy changes are based on the weight of polymer in each specimen.

\section{RESULTS}

\section{Curing time effects}

The finite rate of enthalpy changes $(d H / d t)$ for $13-$ 300-s light-cured UDMA polymer annealed at $37.0^{\circ}$, $57.0^{\circ}$, and $65.5^{\circ} \mathrm{C}$ are plotted against annealing time $(t)$ in Figures 2-4, respectively. Similar results were found for light-cured ZS-filled UDMA and MAPMsilanated ZS-filled UDMA composites tested cured for the same time and measured at the same annealing temperatures. At $57.0^{\circ}$ and $65.5^{\circ} \mathrm{C}$, the changes in $\mathrm{dH} /$ $d t$ for the 13-s light-cured UDMA polymer and

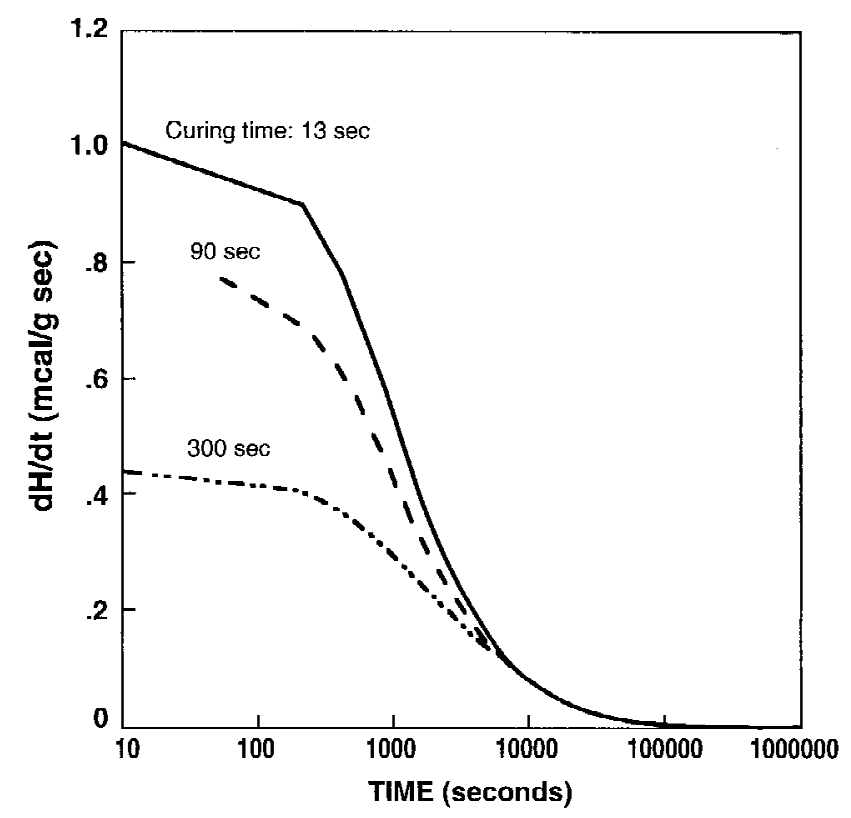

Figure 2. Rate of change of enthalpy (mcal/g s) as a function of time (s) for UDMA polymer cured for different lengths of time and annealed at $37.0^{\circ} \mathrm{C}$.

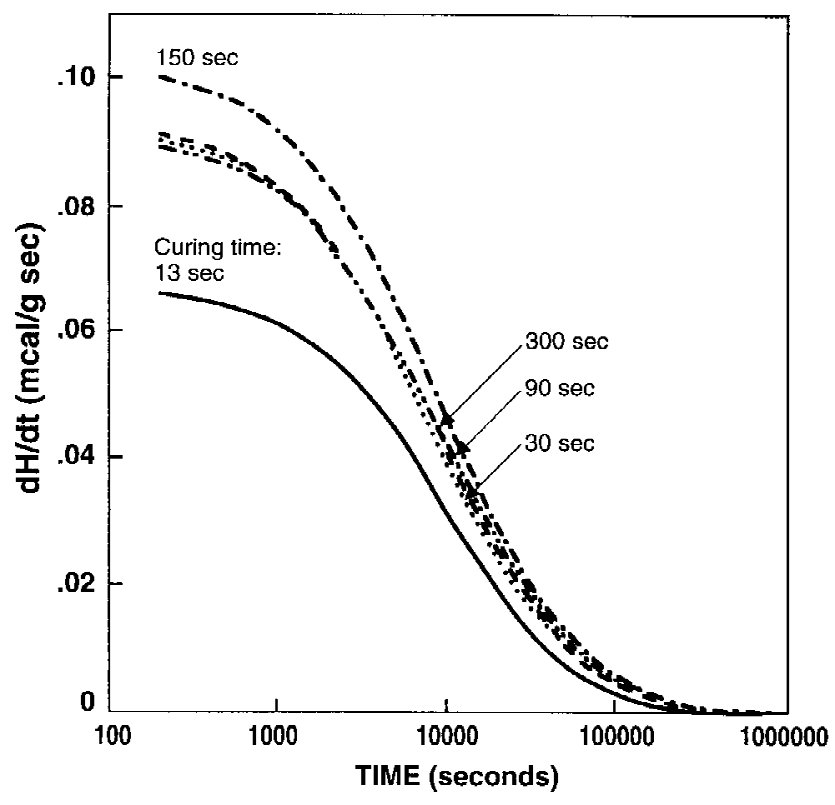

Figure 3. Rate of change of enthalpy (mcal/g s) as a function of time (s) for UDMA polymer cured for different lengths of time and annealed at $57.0^{\circ} \mathrm{C}$.

UDMA-based composite are lower than those for the 300-s light-cured systems at short and long annealing times. Regardless of the annealing temperatures and the composite systems tested, samples cured for longer periods with visible light reached apparent equilibrium faster than those cured for shorter times. For example, the UDMA polymer samples that were cured for $13 \mathrm{~s}$ and annealed at $57.0^{\circ}$ and $65.5^{\circ} \mathrm{C}$ reached equilibrium at $4.5 \times 10^{5}$ and $4.0 \times 10^{5} \mathrm{~s}$, re-

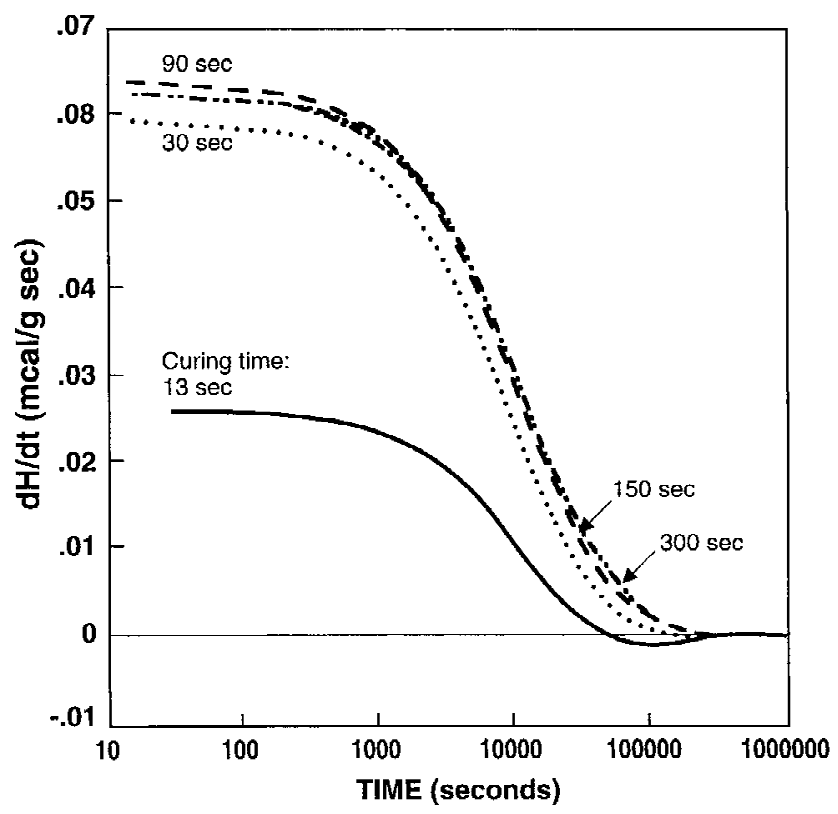

Figure 4. Rate of change of enthalpy (mcal/g s) as a function of time (s) for UDMA polymer cured for different lengths of time and annealed at $65.5^{\circ} \mathrm{C}$. 
spectively, while those cured for $300 \mathrm{~s}$ reached equilibrium at $4.0 \times 10^{5}$ and $2.4 \times 10^{5} \mathrm{~s}$. However, at $37.0^{\circ} \mathrm{C}$, the change in $d H / d t$ was greater for the composite systems cured for $13 \mathrm{~s}$ than those cured for $300 \mathrm{~s}$. In addition, the equilibrium time was reached at $1.0 \times 10^{6}$ $\mathrm{s}$ for UDMA polymer when cured for 13 and $300 \mathrm{~s}$, while it was reached at $3.5 \times 10^{5}$ and $5.5 \times 10^{5} \mathrm{~s}$ for samples containing $75 \mathrm{wt} \%$ filler and cured for 13 and $300 \mathrm{~s}$, respectively. Further, the equilibrium time was $5.0 \times 10^{5}$ and $1.0 \times 10^{6} \mathrm{~s}$ for samples containing silanated filler when cured for 13 and $300 \mathrm{~s}$, respectively.

The $d H$ for UDMA polymer cured for $13-300 \mathrm{~s}$ and annealed at $65.5^{\circ} \mathrm{C}$ were calculated and are plotted against $t$ in Figure 5. The data shown are for single runs; however, duplicate runs resulted in values that had errors of $1-10 \%$. At short curing times (13 and 30 s), the samples absorbed heat at longer annealing times; this result is in contrast to samples at low annealing temperatures $\left(37.0^{\circ}\right.$ and $\left.57.0^{\circ} \mathrm{C}\right)$. In addition, the composites that contained silanated filler showed similar behavior. Further, at $65.5^{\circ} \mathrm{C}$, samples cured for longer times with visible light had greater enthalpy changes than samples cured for shorter times. Also, the initial measurable $d H$ (i.e., $d H$ at $t=3.3,2.5$, and 0.5 $\mathrm{h}$ for $65.5^{\circ}, 57.0^{\circ}$, and $37.0^{\circ} \mathrm{C}$, respectively) increased with increasing curing time of composites.

To further illustrate the curing time effect on enthalpy changes, $d H$ obtained at $0,10^{4}$, and $10^{5} \mathrm{~s}$ for $0-75 \mathrm{wt}$ $\%$ ZS-filled UDMA and MAPM silanated ZS-filled UDMA composite are plotted against curing time in Figure 6 at $65.5^{\circ} \mathrm{C}$. Similar results were found when the samples were annealed at $57.0^{\circ}$ and $37.0^{\circ} \mathrm{C} . d H$ values increased within increasing curing times and

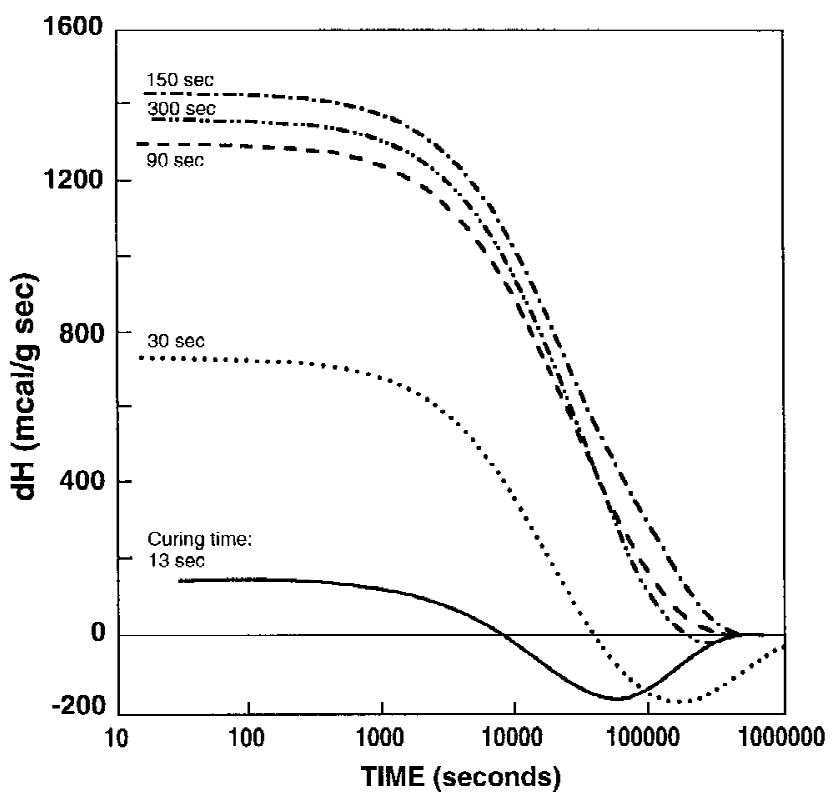

Figure 5. Rate of change of enthalpy (mcal/g) as a function of time (s) for UDMA polymer cured for different lengths of time and annealed at $65.5^{\circ} \mathrm{C}$.

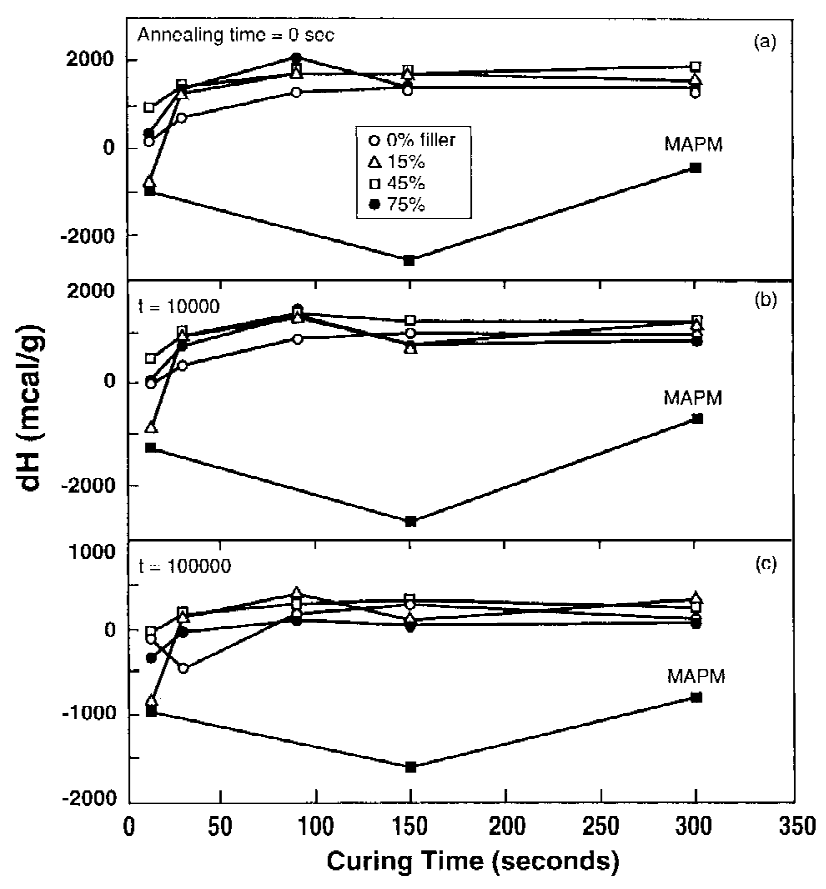

Figure 6. Change of enthalpy (mcal/g) as a function of curing time (s) for 0, 15, 45, and 75 wt \% ZS-filled UDMA and MAPM-silanated ZS-filled UDMA annealed at $65.5^{\circ} \mathrm{C}$ for (a) $0 \mathrm{~s}$, (b) $10^{4} \mathrm{~s}$, and (c) $10^{5} \mathrm{~s}$.

gradually leveled off to constant values at very high curing times when the composites contained unsilanated filler. However, for composites containing silanated filler, the $d H$ values reached a minimum and then increased with longer curing time. $t_{\max }$ was determined as the time when the thermovoltage became constant.

Relative conversion for UDMA annealed at $57.0^{\circ} \mathrm{C}$ is plotted as a function of normalized time in Figure 7. Similar results were found for the filled systems. Regardless of the system tested, the conversion with normalized time was greater for samples cured for short exposures to visible light. For example, in the initial $10 \%$ of the annealing period, the relative conversion of the 13-s cured UDMA polymer was $75 \%$, while the relative conversion of the 300-s cured polymer was $55 \%$. In addition, within $50 \%$ of the annealing period, the conversions were 97 and $90 \%$ for UDMA polymer cured for 13 and $300 \mathrm{~s}$, respectively.

\section{Filler effects}

The $d H / d t$ for 0-75 wt \% ZS-filled UDMA and MAPM-silanated ZS-filled UDMA samples cured with visible light for $13 \mathrm{~s}$ and annealed at $57.0^{\circ} \mathrm{C}$ are plotted against $t$ in Figure 8. All the data for filled samples are normalized to the amount of polymer. Similar results were obtained for these systems at the different an- 


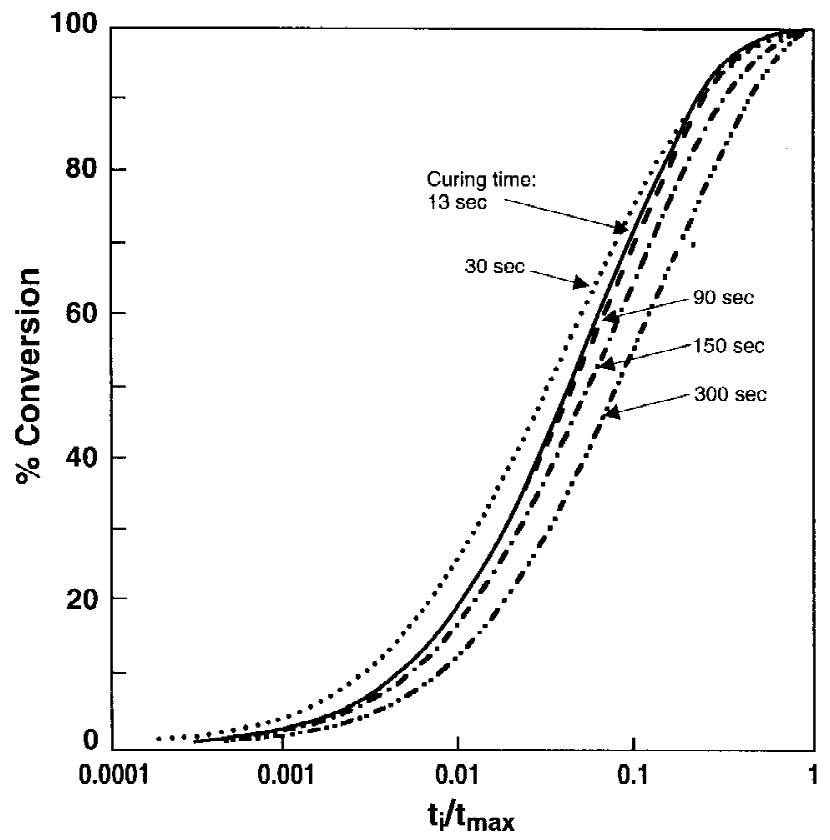

Figure 7. Percent conversion as a function of normalized annealing time for UDMA polymer cured for 13-300 s and annealed at $57.0^{\circ} \mathrm{C}$.

nealing temperatures. The change of $d H / d t$ increased with higher filler concentration in the composites, while the time required to reach apparent equilibrium decreased. For example, at $57.0^{\circ} \mathrm{C}$, the rate of $d \mathrm{H} / \mathrm{dt}$ increased from $2.2 \times 10^{-6}$ to $3.5 \times 10^{-6}$ for filler incorporation from 0 to $75 \mathrm{wt} \%$ when the composite was cured for $13 \mathrm{~s}$, while the apparent equilibrium time decreased from $4.5 \times 10^{5}$ to $1.1 \times 10^{5} \mathrm{~s}$. In addition, the

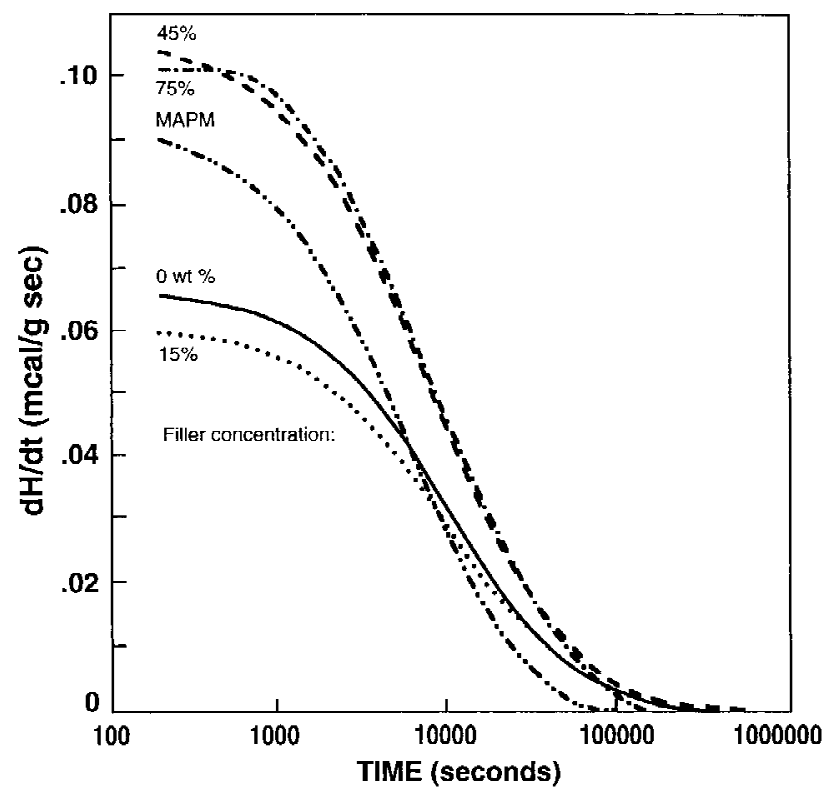

Figure 8. Rate of change of enthalpy (mcal/g s) as a function of time (s) for UDMA-based composites containing 0-75 wt \% unsilanated filler and 75 wt \% MAPM-silanated filler which were cured for $13 \mathrm{~s}$ and annealed at $57.0^{\circ} \mathrm{C}$. rate of change of $d H / d t$ increased in samples consisting of composites containing silanated filler, and the time required to reach equilibrium also decreased compared to composites containing unsilanated filler.

To illustrate the effect of filler concentration on the enthalpy changes, $d \mathrm{H}$ obtained at $0,10^{4}$, and $10^{5} \mathrm{~s}$ for samples cured for different times and annealed at $65.5^{\circ} \mathrm{C}$ are plotted against filler concentration in Figure 9. These data showed nonlinear dependence of the change of enthalpy with filler concentration. The $d H$ curves showed maxima for samples containing $25 \mathrm{wt}$ $\%$ filler at high annealing temperatures, while the $d H$ values increased linearly with the greater filler concentration at low annealing temperatures.

The conversion of 0-75 wt \% ZS-filled UDMA and MAPM-silanated ZS-filled UDMA cured for $13 \mathrm{~s}$ and annealed at $57.0^{\circ} \mathrm{C}$ are plotted as a function of normalized time in Figure 10. Greater filler concentration increased the relative conversion from 75 to $85 \%$ for 0 and $75 \mathrm{wt} \%$ ZS-filled UDMA, while silanating the filler caused a decrease in the conversion to $66 \%$ when the samples were annealed at the initial $10 \%$ of the time.

\section{Temperature effects}

The $d H / d t$ for $75 \mathrm{wt} \%$ ZS-filled UDMA annealed at $37.0^{\circ}, 57.0^{\circ}$, and $65.5^{\circ} \mathrm{C}$ and cured for $13 \mathrm{~s}$ are plotted against $t$ in Figure 11. Similar results were obtained for

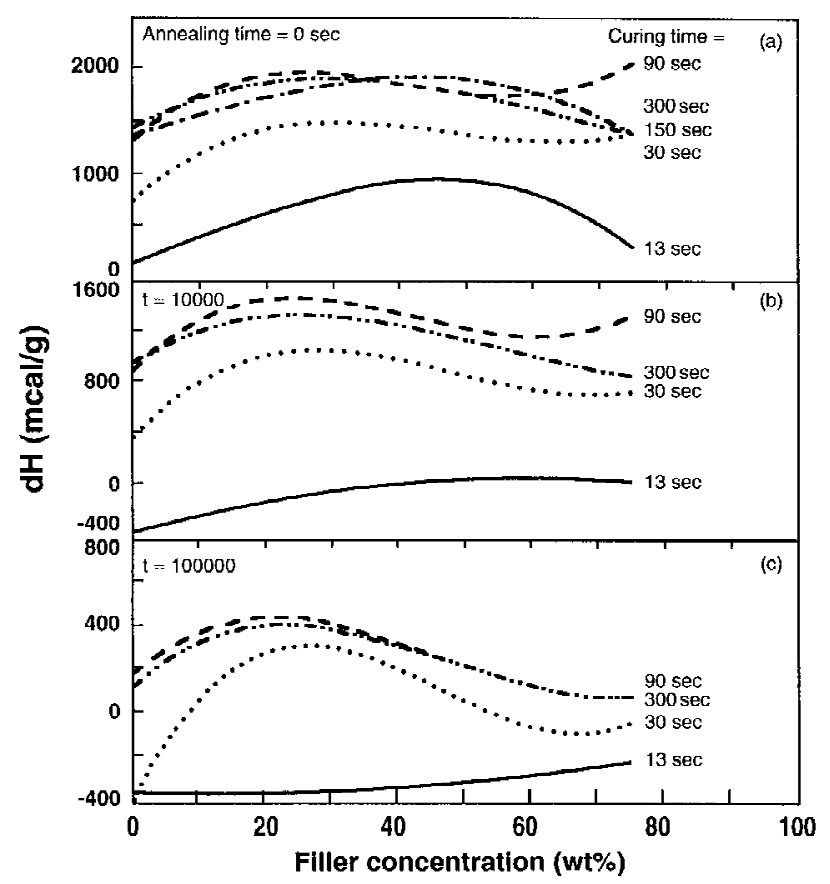

Figure 9. Change of enthalpy (mcal/g) as a function of filler concentration (wt \%) for 13-300-s cured ZS-filled UDMA annealed at $65.5^{\circ} \mathrm{C}$ for (a) $0 \mathrm{~s}$, (b) $10^{4} \mathrm{~s}$, and (c) $10^{5} \mathrm{~s}$. 


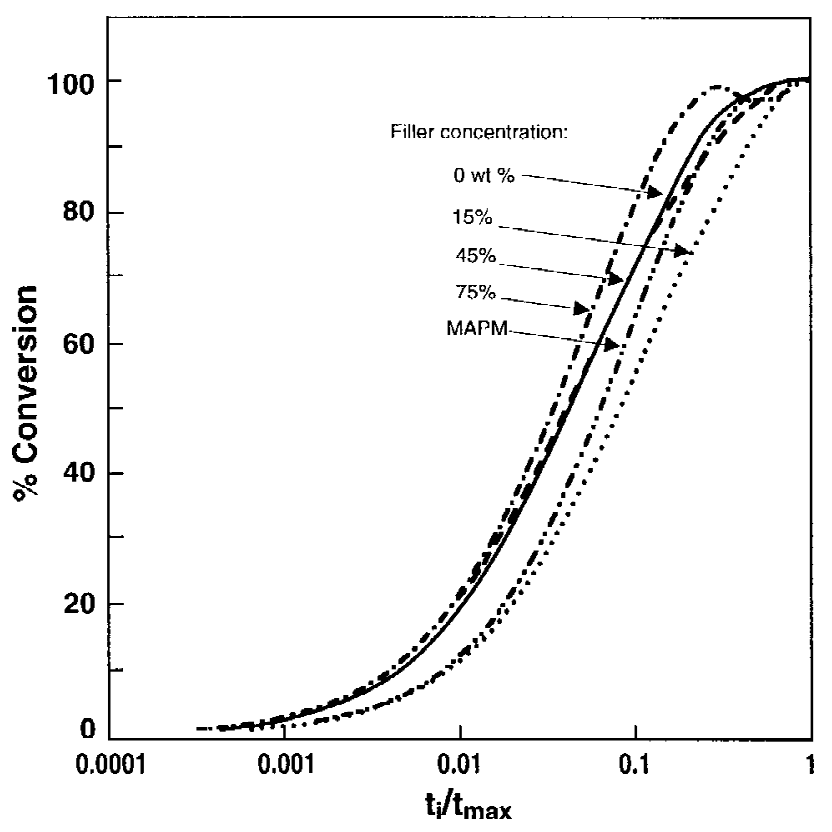

Figure 10. Percent conversion as a function of normalized annealing time for 0-75 wt \% ZS-filled UDMA and MAPMsilanated ZS-filled UDMA cured for $13 \mathrm{~s}$ and annealed at $57.0^{\circ} \mathrm{C}$.

unfilled and silanated ZS-filled samples cured for different curing times. The initial values of $d H / d t$ and the rate of change of $d H / d t$ were higher at the lower annealing temperatures and lower curing times. Also, the time needed for the 75 wt \% ZS-filled UDMA samples to reach apparent equilibrium were $5.5 \times 10^{5}$, $2.1 \times 10^{5}$, and $1.5 \times 10^{5} \mathrm{~s}$ at $37.0^{\circ}, 57.0^{\circ}$, and $65.5^{\circ} \mathrm{C}$,

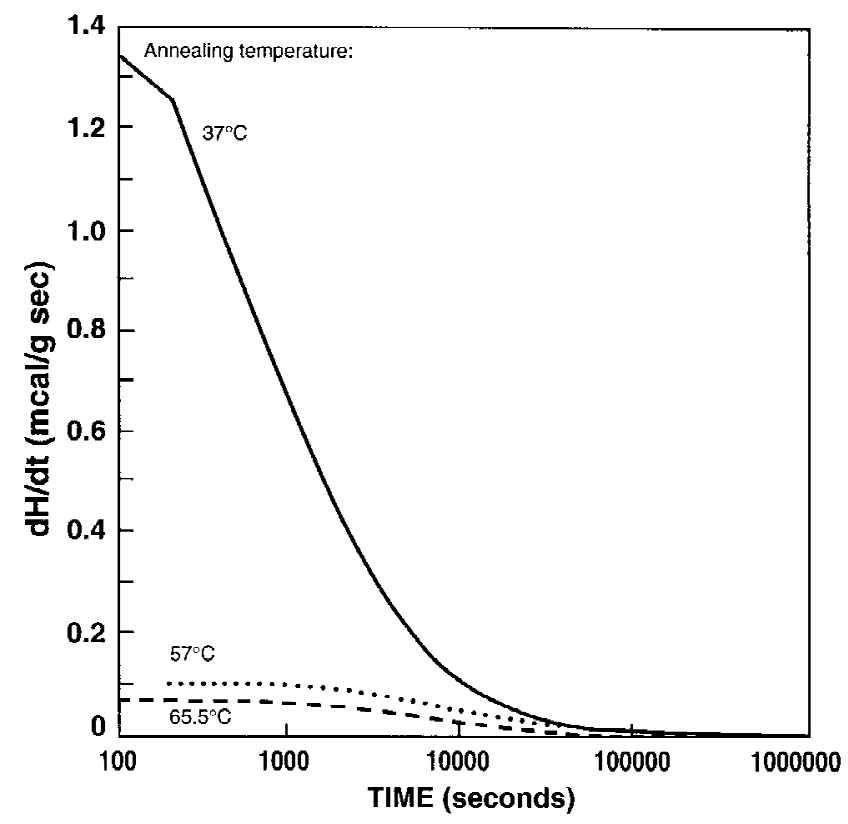

Figure 11. Rate of change of enthalpy (mcal/g s) as a function of time (s) for $75 \mathrm{wt} \%$ ZS-filled UDMA cured for $13 \mathrm{~s}$ and annealed at $37.0^{\circ}, 57.0^{\circ}$, and $65.5^{\circ} \mathrm{C}$.

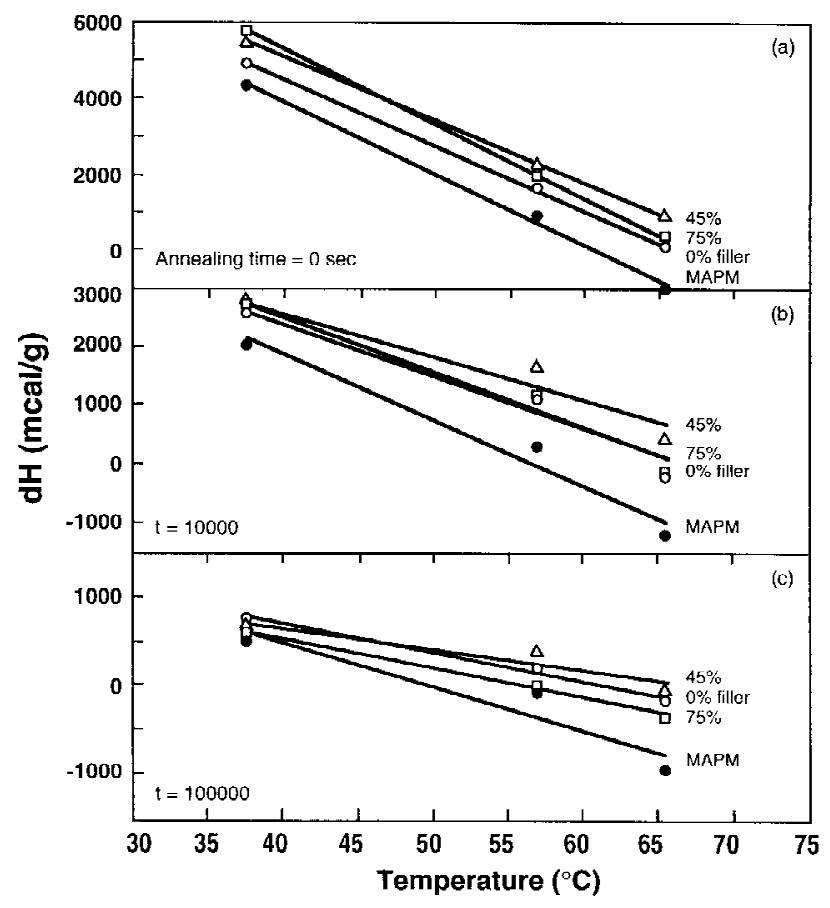

Figure 12. Change of enthalpy (mcal/g) as a function of annealing temperatures $\left({ }^{\circ} \mathrm{C}\right)$ for $13 \mathrm{~s}$ cured 0,45 , and $75 \mathrm{wt}$ $\%$ ZS-filled UDMA and 75 wt \% MAPM-silanated ZS-filled UDMA annealed for (a) $0 \mathrm{~s}$, (b) $10^{4} \mathrm{~s}$, and (c) $10^{5} \mathrm{~s}$.

respectively, when cured for $300 \mathrm{~s}$. However, the time required to reach equilibrium for composites containing silanated filler was independent of the annealing temperatures, and it took $1.0 \times 10^{6} \mathrm{~s}$.

To illustrate further the effect of annealing temperature on the enthalpy changes, the enthalpies for the different samples cured for $13 \mathrm{~s}$ were obtained at 0 , $10^{4}$, and $10^{5} \mathrm{~s}$ of annealing are plotted against annealing temperatures in Figure 12. These data showed a decrease in enthalpy change with higher annealing temperature. In addition, the $d H$ values showed a linear dependence with annealing temperature; thus, the specific heats of the different systems were calculated from the slopes of these curves at $0 \mathrm{~s}$ annealing time, and the values are presented in Table I. The specific heats decreased with longer curing times in UDMA polymer and in the composite containing silanated

TABLE I

Specific Heat (mcal/g ${ }^{\circ} \mathrm{C}$ ) for Different Composite Systems Cured for 13, 90, and $300 \mathrm{~s}$

\begin{tabular}{lccc} 
& \multicolumn{3}{c}{ Specific Heat $\left(\mathrm{mcal} / \mathrm{g}{ }^{\circ} \mathrm{C}\right)$} \\
\cline { 2 - 4 } & \multicolumn{3}{c}{ Curing Time (s) } \\
\cline { 2 - 4 } \multicolumn{1}{c}{ Filler $($ wt $\%)$} & 13 & 90 & 300 \\
\hline 0 & 172 & 120 & 108 \\
75 unsilanated & 200 & 200 & 200 \\
75 MAPM silanated & 192 & & 100 \\
\hline
\end{tabular}


filler, and increased with greater filler concentration, reaching a constant value regardless of the effect of curing time.

The conversions of 75 wt \% ZS-filled UDMA annealed at different temperatures and cured for $13 \mathrm{~s}$ are plotted as a function of normalized time in Figure 13. Similar results were obtained for all filled and unfilled systems. The conversion of the composite was greater with higher annealing temperatures. Also, there was an interaction between the effects of curing times and the annealing temperatures. That is, at very short curing times and high temperatures, a maximum appeared in the conversion curves, indicating the formation of some complexes between the UDMA resin and other extraneous materials present in the system. These agents are possibly hydrogen-bond donor molecules such as moisture or tertiary amine accelerator.

\section{DISCUSSION}

The crosslinking reaction of thermosets is a highly exothermic process, and the heat liberated tends to accelerate the curing reaction. The crosslinking of the diacrylate resins is accomplished by addition polymerization reactions, where the carbon double bonds on the acrylate are converted to a single bond. The exothermic heat of polymerization results from the propagation reaction of free radical chains that crosslink to an active double bond of unsaturated monomers. ${ }^{9}$ Therefore, it can be reasonably assumed

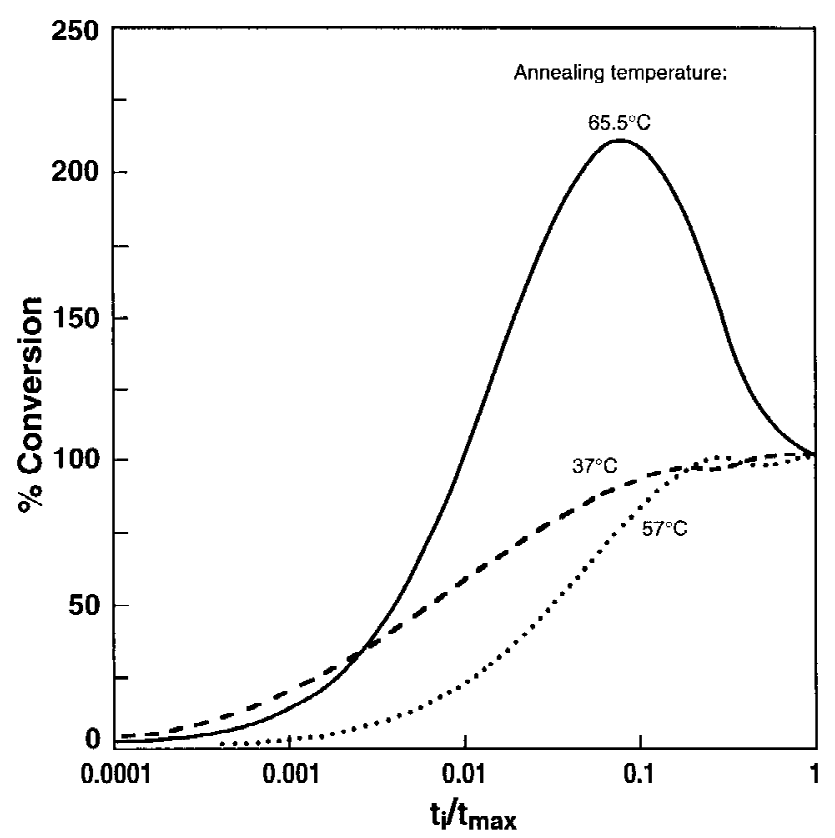

Figure 13. Percent conversion as a function of normalized annealing time for $75 \mathrm{wt} \%$ ZS-filled UDMA cured for $13 \mathrm{~s}$ and annealed at $37.0^{\circ}, 57.0^{\circ}$, and $65.5^{\circ} \mathrm{C}$. that the exothermic heat generated per unit mass and its magnitude can be used to follow the progress of the cure.

From the isothermal measurements, the rates of change of enthalpy and enthalpy changes of the UDMA polymer were found to be exothermic, and they were affected by the curing time, filler content, and nature of the filler surface. The maximum time required for these reactions to reach equilibrium at annealing temperatures of $37^{\circ}-65.5^{\circ} \mathrm{C}$ was $10^{6} \mathrm{~s}$.

\section{Curing time effect}

The microcalorimetry measurements indicate that UDMA polymer and UDMA-based composites cured for longer periods of time with visible light always had higher rates of enthalpy and reaction heats than those cured for shorter periods. This result suggests that annealing of resins cured for shorter times did not increase the crosslink density with time compared to resins cured for longer times, thus leaving more reacted double bonds at the end of the curing and postcuring cycle and lower crosslink density. This slowing down of the curing process is due to reaching either the gel or vitrification point, which results in slowing the diffusion of unreacted double bonds. Thus, this effect resulted in longer times required to reach equilibrium (Figs. 3 and 4) and lower heats of reaction (Fig. 6).

At short curing times (13 and $30 \mathrm{~s}$ ) and high annealing temperatures $\left(65.5^{\circ} \mathrm{C}\right)$ for the UDMA and UDMAbased composites, the rates of enthalpy and the enthalpy changes exhibited an endothermic region at a long annealing time. This observation suggests that in addition to autoacceleration, a reaction occurred between the UDMA resin and other materials present in the system. These agents are possibly hydrogen-donor molecules which may be present in the system such as moisture. ${ }^{10}$ Also, at these short curing times the reaction of the reducing agent and the camphoroquinone to form an exited complex state (exiplex) might not be complete, and the endothermic reaction may be a result of the reduction of the photoinitiator to form free radicals. In addition, it appeared that curing for 150 $300 \mathrm{~s}$ had no significant effect on the enthalpy changes, especially at low filler concentration, which suggests that curing for more than $90 \mathrm{~s}$ with visible light did not change the crosslink density.

Although the final conversion of the partially cured UDMA polymer and the UDMA-based composites did not reach the high conversion of those of the higher cured polymer, the conversion rates were higher at the normalized time for the lower cured polymer and composite (Fig. 7). This contradiction may be explained by the relative number of carbon 
double bonds converted to single bonds, which is higher in the lower cured composites, because of the lower density of packing, higher diffusion rates of chain segments, and higher availability of carbon double bonds.

\section{Filler effect}

The heat of reaction of the polymer-based composites could be modified by the introduction of a plasticizer, copolymerization, or the introduction of inorganic filler. In this study, the heat of reaction of the UDMA was modified by the introduction of different amounts of filler. This modification was reflected by the increase in the values of the enthalpy changes and their rates when the filler concentration was increased to $25 \%$ (Fig. 9), while the values started to decrease at higher filler concentrations. The increases in these values at low filler concentrations were an indication of an introduction of a phase which restricted the molecular motion of the surface layer of polymer onto filler particles, and regarded the composite as being a densely crosslinked polymer. As a result of the crosslinked structure, the thermoset was farther away from the glassy state and equilibrium, which caused higher enthalpy values. However, at higher filler concentrations, the enthalpy changes and their rates started to decrease. This observation may be explained by the formation of some filler aggregates covered with UDMA owing to their high concentration, ${ }^{11}$ or it may be explained by the effect of less penetration of light which caused lower activation and formation of free radicals. This latter effect may result in lower number of propagating free radicals chains and the presence of higher number of unreacted double bonds, which leads to lower crosslink density.

The relative conversion of the filled-UDMA was higher than the unfilled polymer. This observation indicates that the lower penetration of light due to the presence of the filler in the composite caused greater presence of unreacted double bonds; thus, the relative number of carbon double bonds converting to single bonds is higher in highly filled composites.
In composites containing silanated filler, the crosslink density of the composite increased, as shown by the increase of the rate of change of enthalpy (Fig. 8 ) and the decrease of the apparent time required to reach equilibrium. However, the change of enthalpy for samples containing silanated filler was lower than those of the unsilanated ones. Also, the overall change of enthalpy was endothermic. This observation indicates that there is breaking and forming of bonds in the system, which may be due to the dynamic equilibrium created at the interface between the filler and the polymer, which serves as an internal stress reliever.

\section{References}

1. A. Lee and G. McKenna, "Effect of crosslink density on physical aging of epoxy networks," Polymer, 29, 812-817 (1988).

2. M. B. Mangion and G. P. Johari, "Relaxation of thermosets: III. Sub- $T_{g}$ dielectric relaxation of bisphenol-A-based epoxide cured with different cross-linking agents," J. Polym. Sci. Polym. Phys., 28, 71-83 (1990).

3. E. P. Plueddemann, "Catalytic effect in bonding thermosetting resins to silane-treated fillers," in Fillers and Reinforcements for Plastics, Advances in Chemistry Series, Vol. 134, R. D. Deanin and N. R. Schott (eds.), Washington, DC, American Chemical Society, 1974, pp. 113-126.

4. E. D. Plueddemann and G. L. Stark, "Interface in polymer matrix composites," in Composite Materials, Vol. 6, M. W. Ronney, S. E. Berger, and G. C. Massden (eds.), Academic Press, New York, 1974.

5. C. Shu, "Isothermal enthalpy changes of PS/PP blends," Ph.D. Thesis, University of Michigan, 1992.

6. P. Wu, "Recovery of pressure-densified polystyrene," Ph.D. Thesis, University of Michigan, 1989.

7. N. M. Mohsen and R. G. Craig, "Effect of silanation of filler on their dispersibility by monomer systems," J. Oral Rehab., 22, 183-189 (1995).

8. W. H. Douglas, R. G. Craig, and C. C. Chen, "A new composite restorative based on a hydrophobic matrix," J. Dent. Res., 58, 1981-1985 (1979).

9. S. Y. Pusatcioglu, A. L. Fricke, and J. C. Hassler, "Heats of reaction and kinetics of a thermoset polyester," J. Appl. Polym. Sci., 24, 937-946 (1979).

10. A. Dutta and M. E. Ryan, "Effect of fillers on kinetics of epoxy cure," J. Appl. Polym. Sci., 24, 635-649 (1979).

11. Y. U. Lipatov, V. F. Rosovizky, and V. V. Shifrin, "Viscoelastic properties of the model system: Epoxy resin-glass beadspoly(buthylmethacrylate)," J. Appl. Polym. Sci., 27, 455-460 (1982). 